\title{
EDITORIAL
}

....early signs of change are slowly emerging.

\section{Will cancer drugs get cheaper in 2020?}

\author{
Dramatic reductions in drug costs in 2020 are unlikely, although early signs suggest that the issue \\ of unsustainable drug pricing is beginning to attract the attentions of policymakers.
}

The issue of cancer drug pricing requires very little introduction. Suffice to say, for most patients and health-care providers, novel anticancer drugs are either already unaffordable or becoming so. Although this is a global problem, nowhere is this currently more of an issue than in the USA, which has some of the highest drug prices in the world.

Reflecting the notion that drug prices are becoming unsustainable, several attempts to legislate against high health-care costs are currently in progress. On 12 December 2019, the Lower Drug Costs Now Act ${ }^{1}$, a bill expected to reduce federal government spending on health-care by an estimated US $\$ 92$ billion over 10 years, was passed by the House of Representatives. This bill, if enacted, will enable the government to negotiate the prices of 250 medications (including several anticancer drugs), and these negotiated prices would also apply to private insurers; however, this proposal is likely to be rejected by Senate Republicans. This experience highlights a need for bipartisan support if any legislation is to succeed in reducing health-care costs, and several alternative approaches are currently under consideration. Indeed, another bill, the Prescription Drug Pricing Reduction $\mathrm{Act}^{2}$, with the more modest aims of reducing out-of-pocket spending and restricting further increases in drug prices for Medicare beneficiaries, has passed the Senate Finance Committee. Regardless of whether one of these Bills should ultimately be enacted, patients are unlikely to see realistic changes in 2020 - for example, the Lower Drug Costs Now Act has a planned implementation timeline commencing in 2023. Moreover, the pharmaceutical industry will likely seek to recover any financial shortfalls caused by lower drug prices, and this might have important implications for drug prices in other health-care systems.

Critics of government restrictions on drug prices often cite the notion that, in countries with a free-market economy, the cost of health care should reflect what the market will bear. Indeed, in 2019, following the FDA approval of entrectinib for patients with NTRK-fusionpositive solid tumours (and ROS1-mutant non-small-cell lung cancer), the manufacturer declared a US price of approximately half that of the previously approved similar agent, larotrectinib. This price has been described by some observers as a ' $50 \%$ discount' ${ }^{3}$. Nonetheless, few would argue that a price of $\$ 17,050$ per month will enable access for all eligible patients. Moreover, an expectation of similar 'price war' effects among other on-patent agents seems unrealistic, given the historical approach to cancer drug pricing.

A limited period of patent protection and/or market exclusivity is a key feature of legislation relating to new drugs. This implies that outside of these protections, other manufacturers would then be able to produce generic forms of a drug with a more competitive price. In 2019, the FDA took several measures to encourage transparency, including in determining which drugs are off patent and/or market exclusivity but nonetheless lack a generic competitor, and clarifying which drugs are eligible for access restrictions (a loophole often used by pharmaceutical companies to delay the development of generic forms of off-patent drugs). In the same year, the FDA announced full approvals of 971 generic drugs. This total included 125 new generics across all indications, including generic forms of everolimus, carfilzomib, valrubicin and fulvestrant ${ }^{4}$. Did these result in substantial cost savings? For these examples, it might be too early to say. However, if pricing of these drugs reflects the experience with generic imatinib, initial savings will likely be modest. As an example, the manufacturer of the first generic version of imatinib launched their new agent in the USA at a price of around $\$ 60,000$ per year, or approximately $30 \%$ less than the nongeneric form of this drug. Although prices vary across health systems, the expected substantial price decrease as more generic versions of imatinib enter the market has, thus far, largely failed to materialize ${ }^{5}$. The situation for generic forms of biologic agents (biosimilars) is more challenging owing to the much more complicated manufacturing processes.

In conclusion, the costs of most anticancer drugs are unlikely to decrease in 2020 , and any reductions in price are unlikely to be substantial. Nonetheless, early signs of change are slowly emerging.

1. U.S. Congress. H.R.3 - Elijah E. Cummings Lower Drug Costs Now Act. congress.gov https://www.congress.gov/bill/116th-congress/ house-bill/3/actions? KWICView=false (2019).

2. U.S. Congress. S.2543 - Prescription Drug Pricing Reduction Act of 2019. congress.gov https://www.congress.gov/bill/116th-congress/ senate-bill/2543 (2019).

3. Maddipatla, M. Roche cancer treatment priced at $\$ 17,050$ a month, lower than rival Vitrakvi. reuters.com https://www.reuters. com/article/us-roche-hldg-fda-pricing/roche-cancer-treatment-pricedat-17050-a-month-lower-than-rival-vitrakvi-idUSKCN 1 V61 G8 (2019).

4. FDA. FDA Drug Competition Action Plan. fda.gov https://www.fda gov/drugs/guidance-compliance-regulatory-information/fda-drugcompetition-action-plan (2019)

5. Cohen, J. The curious case of Gleevec pricing. forbes.com https:// www.forbes.com/sites/joshuacohen/2018/09/12/the-curious-caseof-gleevec-pricing/\#5e74fb7354a3 (2018). 